\title{
An algorithmic approach to multi-layer wrinkling
}

\author{
Emma Lejeune, Ali Javili, Christian Linder* \\ Department of Civil and Environmental Engineering, Stanford University, Stanford, CA 94305, USA
}

\begin{abstract}
Wrinkling, when a thin stiff film adhered to a compliant substrate deforms sinusoidally out of plane due to compression, is a well understood phenomenon in bi-layer systems. However, when there are more than two layers, the wrinkling behavior of the multi-layer system is, at present, not fully understood. In this paper, we provide an analytical solution for wrinkling in tri-layer systems where the additional layers can contribute to either the film stiffness or substrate stiffness. Then, we provide an algorithmic approach for extending our tri-layer analytical solution to systems with multiple additional layers. Our analytical solution and algorithmic approach are verified numerically using the finite element method. Using our methodology, wrinking can be predicted and controlled in multi-layer systems, with applications ranging from stretchable electronics to biomimetic design. In this paper, we demonstrate that our model can be used to understand wrinkling behavior in epidermal electronics.
\end{abstract}

Keywords: Wrinkling, Geometric instability, Epidermal electronics

\section{Introduction}

Though wrinkling in bi-layer systems is a well understood phenomenon [1-7], the study of wrinkling in multi-layer systems is incomplete. Multi-layer systems, as illustrated in Fig. 1, do not necessarily have a single layer clearly defined as the film or a single layer clearly defined as the substrate. Instead, there are multiple layers some of which may contribute to the axial and bending stiffness of the film and some of which may contribute to the stiffness of the substrate [8-10]. Understanding wrinkling in multi-layer systems is important for interpreting the wrinkling behavior of some biological systems, such as the gut [11-13], the skin [14-16], or the lungs [17, 18]. The patterns which emerge from these biological multi-layer systems can inspire engineering design, such as engineering surfaces which utilize wrinkling to access multiple length scales and increase surface area [19.-21]. In buckling based metrology, a better understanding of wrinkling in multi-layer films will enhance the study of novel multi-layer systems [22-25]. In addition, in engineering stretchable electronics, a deeper understanding of multi-layer wrinkling can be used to design systems in which wrinkling

*Corresponding author.

E-mail address: linder@stanford.edu (C. Linder)

Preprint submitted to Extreme Mechanics Letters is used to prevent high levels of strain in stiff or brittle layers [26, 27]. In this paper, we use epidermal electronics [28-32] as an example to motivate the study of multi-layer wrinkling. Epidermal electronics are a strong motivation because, as seen in Fig. 2, they connect to multi-layer wrinkling in two ways. First the electronic devices themselves are often multi-layer systems [28, 33]. Second, the skin is a multi-layer structure thought to have as many as six mechanically distinct layers which influence its wrinkling behavior [34, 35]. We present a multi-layer model suitable for capturing the wrinkling behavior of the skin-device system.

With regard to previous research specific to multi-layer wrinkling [8], we primarily build on four previous works to construct our approach to multi-layer wrinkling. First, Stafford et al. [37] proposed a method for combining thin, experimentally observed, surface layers of finite thickness with the film layer by treating the film as a composite beam. Second, Jia et al. [9] provided an analytical solution verified by numerical results to account for an intermediate layer that will combine with either the film or the substrate depending on the geometric and material properties of the system. Third, Huang et al. [38] demonstrated that after a finite substrate depth is reached, substrate depth no longer influences wrinkling behavior. Finally, in our 


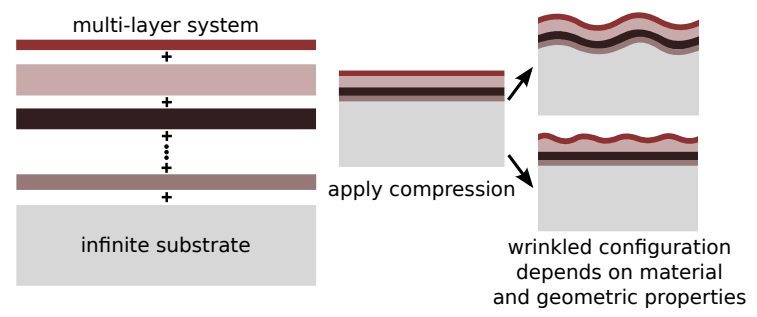

Figure 1: When thin stiff films adhered to compliant substrates experience in plane compression, the wrinkling instability can occur. Though wrinkling in systems that consist of a single film on a substrate is well understood, wrinkling in systems with multiple layers has still not been sufficiently studied. In this paper we provide a general methodology for studying wrinkling in multi-layer systems.

recent work [10] we proposed an analytical solution verified by numerical results to account for wrinkling in multi-layer systems where the additional interfacial layers are assumed to be thinner than the film.

Here, we extend the solution presented in Lejeune et al. [10], which is restricted to bi-layer systems with additional thin interfacial layers, to systems with multiple layers that are not restricted in thickness or material properties. Then, we apply our extended multi-layer wrinkling model to a device-skin system. The rest of the paper is organized as follows. In Section 2 , we clearly explain the methodology for performing this extension with an analytical solution verified by numerical results. In Section 3, we show an example of our multi-layer wrinkling model applied to epidermal electronics. Finally, we conclude our paper in Section 4 .

\section{Multi-layer model}

The equations for critical strain $\varepsilon_{\mathrm{cr}}$, critical wavelength $\lambda_{\mathrm{cr}}$, and critical wave number $n_{\mathrm{cr}}$ that are sufficient to describe wrinkling initiation in a bi-layer system are quite simple. Our intention is to preserve this simplicity by extending the bi-layer solution to multiple layers following an intuitive procedure. We begin with the equation relating axial strain $\varepsilon$ to wave number $n$ used to derive $n_{\mathrm{cr}}$ for bi-layer wrinkling [1, 39],

$$
E_{f} \frac{t_{f}^{3}}{12} n^{4}-E_{f} t_{f} \varepsilon n^{2}=-\frac{2 E_{s}}{\zeta} n
$$

where $E_{f}$ and $E_{s}$ refer to the plane strain moduli of the film and substrate respectively, $v_{s}$ is the Poisson ratio of the substrate, $t_{f}$ is film thickness, and $\zeta=$ $\left(3-4 v_{s}\right) /\left(1-v_{s}\right)^{2}$. In the typical applications related to the wrinkling instability, corresponding to nearly incompressible material behavior, $v_{s} \simeq 0.5$, therefore we will proceed with $\zeta=4$. The term on the right of the equation can be understood as the influence of the infinite substrate on the film. We define $t_{t}$ as the "test" depth used to determine how many layers below the film will contribute to film stiffness given the behavior of the system at instability initiation. If we think of the substrate contribution as a set of springs in parallel, with spring stiffness $K=E_{s} / t_{t}, t_{t}$ is understood as the depth of substrate felt by the film. Given (1), the value of $n_{\mathrm{cr}}$ can be computed explicitly by solving $\mathrm{d} \varepsilon / \mathrm{d} n=0$ with respect to $n$, which gives the explicit solution for $t_{t}$ in a bi-layer system as

$$
n_{c r}=\frac{1}{t_{f}} \sqrt[3]{\frac{3 E_{s}}{E_{f}}} \quad \text { and } \quad t_{t}=\frac{2}{n_{\mathrm{cr}}} .
$$

This is consistent with the notion that after a certain substrate depth is reached, the system will behave identically to a system with an infinite substrate [38, 40]. Based on (2), it is clear that a higher $E_{f} / E_{s}$ ratio corresponds to larger values of $t_{t}$.

In the context of wrinkling initiation in multi-layer systems, computing $t_{t}$ is necessary because it will be used to determine which combination of layers contribute to either film or substrate stiffness. We define the terms "FCL" to denote film contributing layers and "SCL" to denote substrate contributing layers. For example, in a tri-layer system where the film on top is stiffer than both lower layers, computing $t_{t}$ with respect to the film and intermediate layer tests if a possible buckling mode is bi-layer wrinkling entirely independent of the lowest layer. When $t_{t}$ is greater than the thickness of the intermediate layer, the bi-layer equations are no longer sufficient to predict wrinkling. Therefore, a solution for wrinkling in a tri-layer system is required. To address this, we demonstrate that the equations presented in Lejeune et al. [10] for a tri-layer model with thin interfacial layers are extensible to systems with thick interfacial layers. With an intermediate layer that acts as a SCL, (1) becomes

$$
E_{f} \frac{t_{f}^{3}}{12} n^{4}-E_{f} t_{f} \varepsilon n^{2}=-\frac{E_{s} n}{n t_{i}\left(E_{s} / E_{i}-1\right)+2}
$$

where $E_{i}$ is the plane strain intermediate layer modulus, $t_{i}$ is the intermediate layer thickness [10]. The implicit equation, $\mathrm{d} \varepsilon / \mathrm{d} n=0$ can be solved for $n_{\mathrm{cr}}$ and subsequently $\varepsilon_{\mathrm{cr}}=\varepsilon\left(n_{\mathrm{cr}}\right)$. For an intermediate layer which 


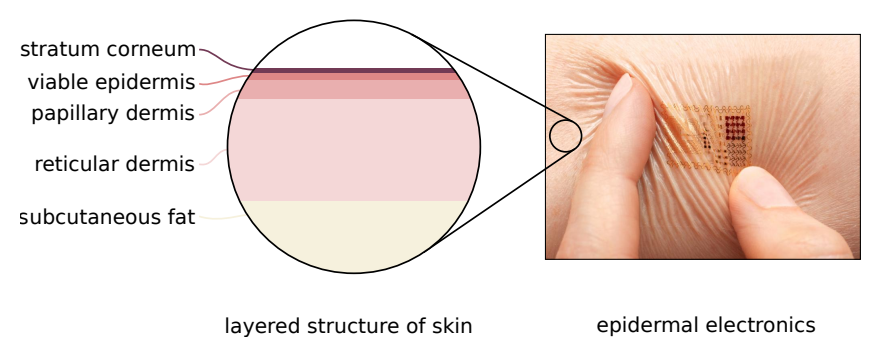

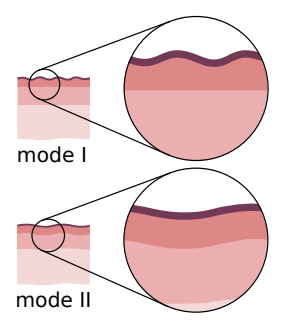

skin wrinkling

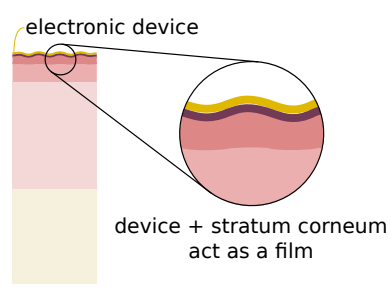

device + skin wrinkling

Figure 2: This figure illustrates the connection between multi-layer wrinkling and epidermal electronics (left center experimental image published in [36]). By modeling the skin with five mechanically distinct layers [35], it is possible to obtain a better understanding of its wrinkling behavior. We find that in the skin-device system the skin and stratum corneum will act as a film while the four lower layers of the skin will act as the substrate. A more detailed analysis is provided in Section 3

contributes to the film stiffness (FCL), the overarching equation is identical to (1), except the bending and axial stiffness of the film must be updated to treat the multilayer film as a composite plate. Equation (1) becomes

$$
\left(E_{i} I_{i}+E_{f} I_{f}\right) n^{4}-\left(t_{i} E_{i}+t_{f} E_{f}\right) \varepsilon n^{2}=-\frac{E_{s} n}{2}
$$

where $I_{i}$ and $I_{f}$ correspond to the moment of inertia divided by width of the intermediate layer and film respectively, computed via the parallel axis theorem. The idea of combining the intermediate layer and film as composite plates was originally proposed in Stafford et al. [37] in the context of buckling based metrology, and the result is a set of explicit equations for $n_{\mathrm{cr}}, \lambda_{\mathrm{cr}}$ and $\varepsilon_{\mathrm{cr}}$.

Given these two options, FCL and SCL, the option which occurs at a lower level of strain will correspond to instability initiation [9]. In Fig. 3, $\varepsilon_{\mathrm{cr}}$ and $\lambda_{\mathrm{cr}}$ for a system with fixed film and substrate properties and varied intermediate layer properties are plotted. It is clearly illustrated that using the bi-layer solution, the SCL tri-layer solution, and the FCL tri-layer solution, instability initiation can be captured throughout the domain. When $E_{i}<E_{s}, t_{t}$ is used to determine if the solution corresponds to the bi-layer or the tri-layer. When $E_{i}>E_{s}$ the correct solution corresponds to a lower value of $\varepsilon_{\mathrm{cr}}$. Based on Fig. 3, it is clear that our extension from a thin intermediate layer to a thick intermediate layer is validated by the good agreement with numerical results.

Like the equations proposed in Jia et al. [9], we are able to capture the entire domain of numerical results. However, for the SCL solution, the equations we use to compute $\varepsilon_{\mathrm{cr}}$ and $\lambda_{\mathrm{cr}}$ are different and somewhat simpler to intuitively understand and implement. Though simpler certainly does not translate to "better", our approach has the benefit of being naturally extensible to systems with more than three layers. With regard to combining multiple intermediate layers to contribute to the substrate, this extension can be conducted by treating the layers as a set of springs in series

$$
t_{i}^{\mathrm{tot}}=t_{k}+t_{k+1} \quad \text { and } \quad E_{i}^{\mathrm{tot}}=\frac{E_{k} E_{k+1}\left(t_{i}+t_{i+1}\right)}{E_{i} t_{i+1}+E_{k+1} t_{k}}
$$

where layers $k$ and $k+1$ are combined to a total layer stiffness, and $t_{\text {tot }}$ and $E_{\text {tot }}$ describe a single layer equivalent to the two layers combined. In order to compute $t_{t}$ when there are intermediate layers contributing to the substrate, we use the value $K$, which represents the substrate contribution given in (3). The value of $K$ is known after implicitly solving $\mathrm{d} \varepsilon / \mathrm{d} n=0$, which means that $t_{t}$ can be computed as

$$
\begin{gathered}
K=\frac{E_{s} n_{\mathrm{cr}}}{n_{\mathrm{cr}} t_{i}\left(E_{s} / E_{i}-1\right)+2} \\
t_{t}=\frac{E_{i} E_{s}-K E_{s} t_{i}}{K E_{i}}
\end{gathered}
$$

To combine multiple layer contributing to film stiffness, the layers are treated as a composite plate [37]. The resulting axial and bending stiffness from combining two layers can be computed as

$$
\begin{gathered}
\bar{y}=\frac{E_{k} t_{k}\left(t_{k+1}+t_{k} / 2\right)+E_{k+1} t_{k+1}\left(t_{k+1} / 2\right)}{E_{k} t_{k}+E_{k+1} t_{k+1}} \\
I_{k}=\frac{1}{12} t_{k}^{3}+t_{k}\left(\bar{y}-\left(t_{k+1}+t_{k} / 2\right)\right)^{2} \\
I_{k+1}=\frac{1}{12} t_{k+1}^{3}+t_{k+1}\left(\bar{y}-t_{k+1} / 2\right)^{2}
\end{gathered}
$$




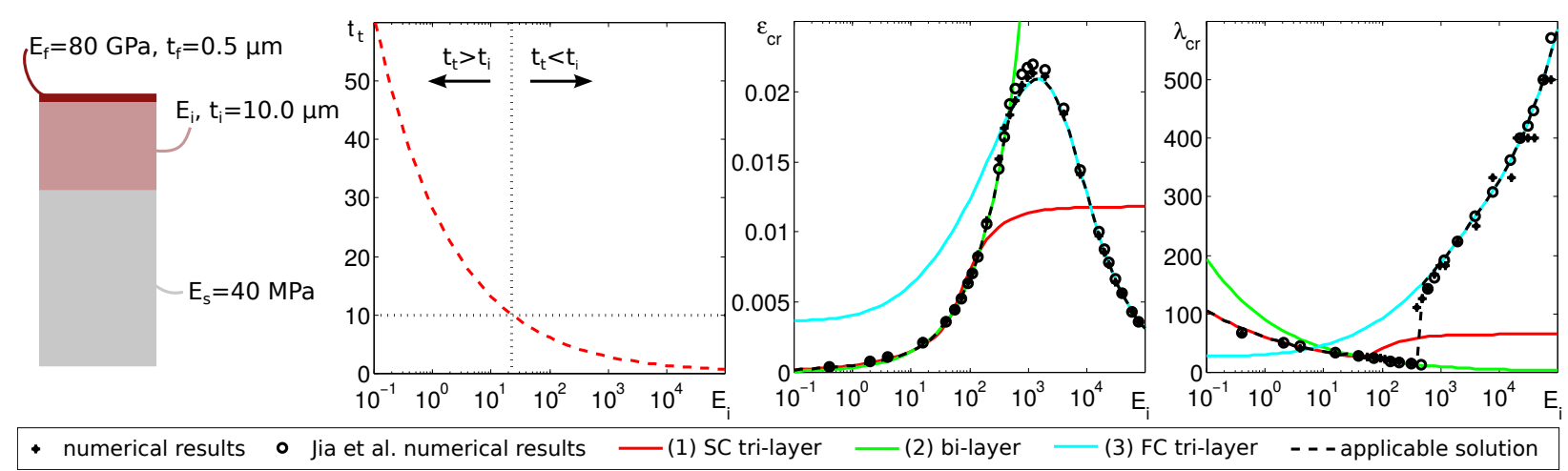

Figure 3: Left: schematic illustration of the system evaluated. Center left: plot of $t_{t}$ given $E_{f}, t_{f}$ and $E_{i}$ to highlight the point when $t_{t}<t_{i}$ and the film no longer feels the lower substrate layer. Center right: plot of $\varepsilon_{\mathrm{cr}} \mathrm{vs}$. $E_{i}$. Right: plot of $\lambda_{\mathrm{cr}}$ vs. $E_{i}$. Overall, the plots shown in this figure confirm that our proposed methodology is capable of handling tri-layer systems with thick intermediate layers. In addition, by comparing our numerical results with the ones present in Jia et al. [9] we show that our methodology for obtaining numerical results is reproducible and sound.

$$
\begin{aligned}
& {[E A]_{\mathrm{tot}}=E_{k} t_{k}+E_{k+1} t_{k+1}} \\
& {[E I]_{\mathrm{tot}}=E_{k} I_{k}+E_{k+1} I_{k+1} .}
\end{aligned}
$$

As with adding subsequent intermediate layers to the substrate, adding additional layers to the film can be conducted iteratively. If $[E A]_{k}^{\text {tot }},[E I]_{k}^{\text {tot }}$ and $\bar{y}_{k}^{\text {tot }}$ corresponding to layer $k$ are known, $[E A]_{k+1},[E I]_{k+1}$ and $\bar{y}_{k+1}$ can be computed readily, then the total values of $[E A]_{k+1}^{\text {tot }},[E I]_{k+1}^{\text {tot }}$ and $\bar{y}_{k+1}^{\text {tot }}$ corresponding to total system $k+1$ are computed as

$$
\begin{gathered}
\bar{y}=\frac{[E A]_{k}^{\mathrm{tot}}\left(\bar{y}_{k}^{\mathrm{tot}}+t_{k+1}\right)+[E A]_{k+1} t_{k+1} / 2}{[E A]_{k}^{\mathrm{tot}}+[E A]_{k+1}} \\
{[E A]_{k+1}^{\mathrm{tot}}=[E A]_{k}^{\mathrm{tot}}+[E A]_{k+1}} \\
{[E I]_{k+1}^{\mathrm{tot}}=\left[\begin{array}{c}
{[E I]_{k}^{\mathrm{tot}}+[E A]_{k+1}^{\mathrm{tot}}\left(\bar{y}-\bar{y}_{k}^{\mathrm{tot}}-t_{k+1}\right)^{2}} \\
+[E I]_{k+1}+[E A]_{k+1}\left(\bar{y}-t_{k+1} / 2\right)^{2} .
\end{array}\right.}
\end{gathered}
$$

By combining (3) and (4), we obtain an expression for a system with a multi-layer film, multi-layer intermediate region and a substrate as

$$
[E I]^{\mathrm{tot}} n^{4}-[E A]^{\mathrm{tot}} \varepsilon n^{2}=-\frac{E_{s} n}{n t_{i}^{\mathrm{tot}}\left(E_{s} / E_{i}^{\mathrm{tot}}-1\right)+2} .
$$

The algorithms provided in Fig. 4 clearly illustrate the procedure for determining which layers contribute to the film and which layers contribute to the substrate such that (9) can be applied. Just as with the bi-layer solution, some systems will not be good candidates for applying the analytical solution and will require numerical analysis [43]. This is particularly the case when the total sum of SCLs is not sufficiently thick, when the conditions of small strain are violated or when different modes of instability may occur at nearly the same level of strain. However, even when obtaining an exact analytical solution is not possible, a qualitative understanding of multi-layer wrinkling may still be helpful [24].

In Fig. 5. multiple different tri-layer configurations are illustrated. Though these small systems can be understood very intuitively and interpreted directly from the equations in Sec. 2, the algorithm presented in Fig. 4 proves useful for more complicated systems. To further demonstrate the validity of our approach for systems with more than three layers, we compare the algorithmic solution for a four layer system to numerical results [43]. The geometry and material properties of the system tested are shown in Fig. 6. In this system, the layers of the substrate becomes stiffer with increasing depth, so the algorithm illustrated in Fig. 4 is used to determine the analytical solution. In the results plotted in Fig. 6 , the properties of the intermediate layers and substrate are held fixed while the film stiffness is increased. As $E_{f}$ increases, the depth of substrate influencing film behavior increases. Therefore, when $E_{f}$ is very low, the first intermediate layer acts as the substrate. When $E_{f}$, and subsequently $t_{s}$, increases, the second intermediate layer and substrate layer both contribute to instability initiation. Based on the good agreement between our numerical and analytical solutions, it is clear that our approach is valid for the system tested. In Fig. 6, the analytical solutions for the bi-layer system of the film and each subsequent layer are also shown to illustrate that while the curves are the same shape, the predicted 


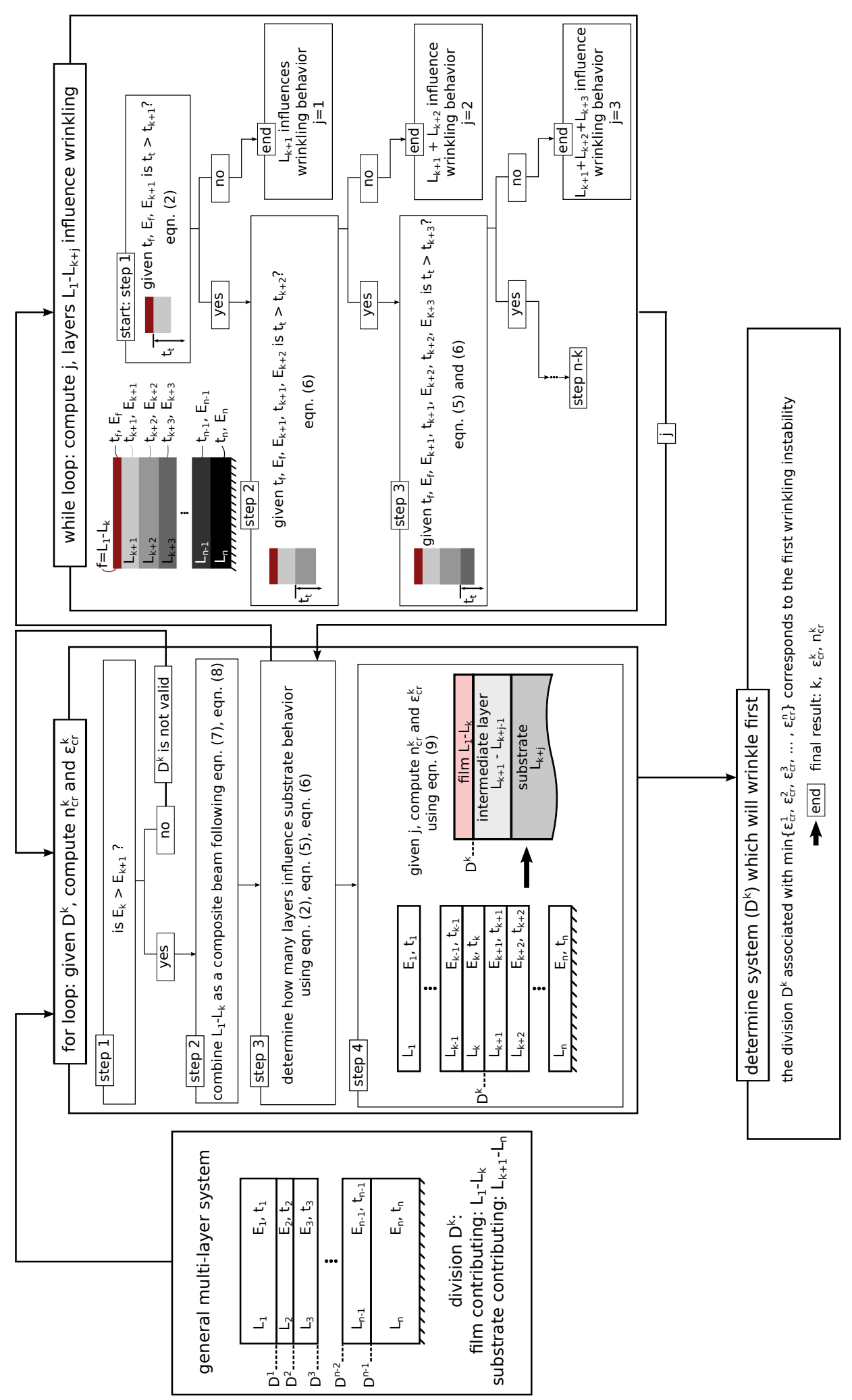

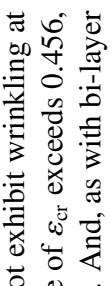

吾闹

है

政

总

孚 을 莎。

四 哭

国

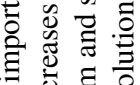

可

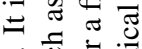

흘

호을

할

要

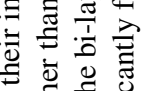

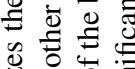

를

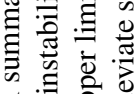

클

部

of

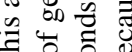

(i)

5

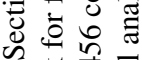

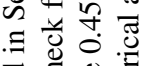

递

잉

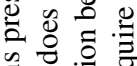

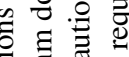

营

D त

足

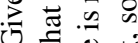

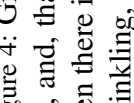

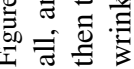



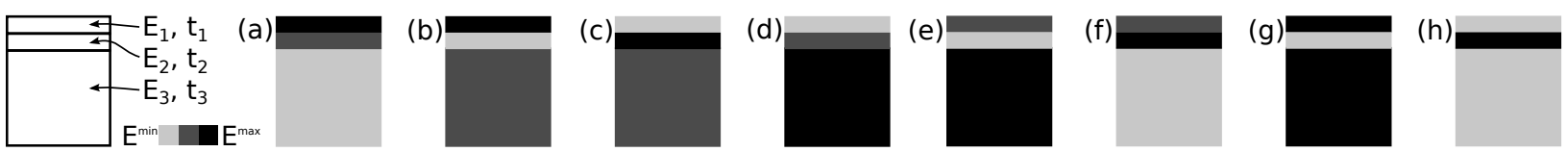

Figure 5: Here all possible tri-layer systems are illustrated. By using the approach outlined in Section 2 these systems can be readily understood. Using the algorithm provided in Fig. 4. wrinkling behavior in each one of these systems can be calculated. As the number of layer increases, predicting system behavior may become more difficult to manage, though the same simple logic is extended.
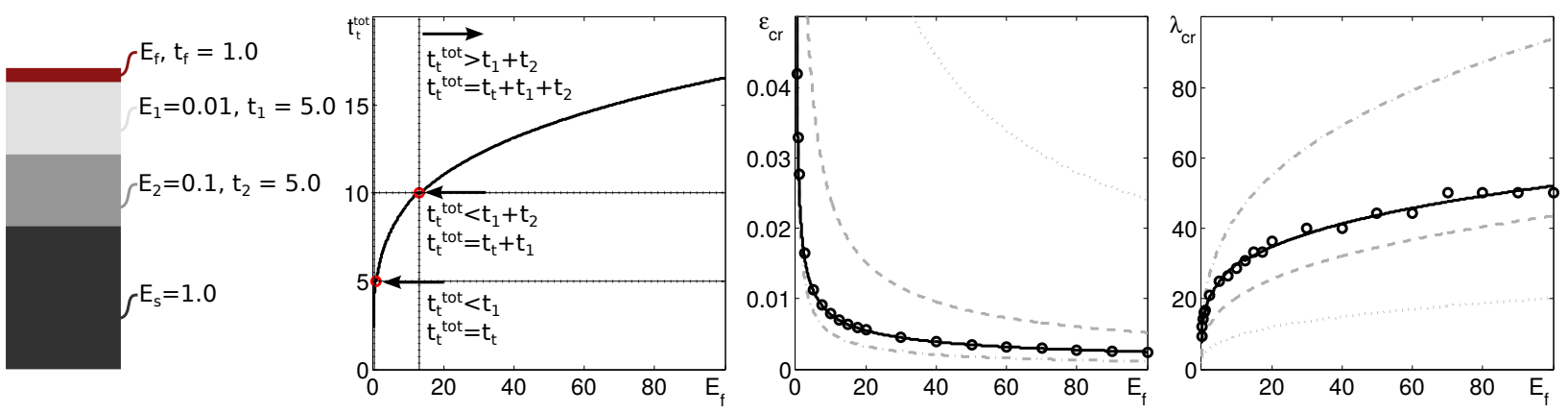

- numerical results -analytical solution

- bi-layer, $\mathrm{E}_{\mathrm{s}}=0.01$

bi-layer, $E_{s}=0.1$ bi-layer, $E_{s}=1.0$

Figure 6: Left: schematic diagram of the system tested analytically and numerically. Left Center: depth below film engaged with respect to different values of $E_{f}$ with a marked transition and additional layers become engaged. Right Center: $n_{\text {cr }}$ plotted with respect to $E_{f}$ for both numerical and analytical results, the incorrect bi-layer solutions are plotted for comparison. Right: $\varepsilon_{\text {cr }}$ plotted with respect to $E_{f}$ for both numerical and analytical results, the incorrect bi-layer solutions are plotted for comparison.

values are sufficiently different such that it is evident that the close agreement between our analytical and numerical solution is not a coincidence.

\section{Application to epidermal electronics}

As illustrated in Fig. 2, images and studies of the human skin indicate that it has a multi-layer structure [44]. Here, we present a model of the skin which has five mechanically distinct layers [35]. Due to the variability in parameters reported in the literature [45-49], both due to differences in experimental set up and variation across subjects and locations on the body [50], the parameters chosen for our model are approximations. Similar to the abrupt mode switch illustrated in Fig. 3. stiffening of skin during the aging process is shown to cause an abrupt switch between mode I wrinkling, where the stratum corneum acts as the film, and mode II wrinkling where the stratum corneum and viable epidermis act as a composite film. With our approach to multi-layer wrinkling, we are able to confirm that this switch is plausible. Previous predictions of this switch, while insightful, relied on very approximate analytical and numerical results [35]. Of course, more accurate quantification of geometry and parameters is required to make quantitative or prescriptive conclusions about wrinkling in the skin. However, this prediction of a mode switch with an abrupt jump in $\lambda_{\text {cr }}$ has been observed experimentally in a study of wrinkling response to applied compression in facial skin for subjects of varied age [51]. Our analysis also indicates that there are multiple systems (combinations of different FCL or SCL) which can potentially wrinkle in the skin, and that an increase in $\varepsilon_{c r}$ may induce longer wavelength wrinkling. For example, wrinkling in the stratum corneum layer may occur first, and then wrinkling of the stratum corneum and viable epidermis acting as a composite film may become superimposed at a higher level of strain. It is also possible that the deformation induced by the lower wrinkling modes will not heavily influence the timing and appearence of the higher modes because the differences in length scale may render wrinkling induced deformation negligible. This is consistent with experimental observations which indicate that multiple superimposed wavelengths may be observed in skin under high levels of compression [14, 16].

The design of epidermal electronics is highly varied and rapidly evolving [28, 30]. Here, we consider a simplified system in which an electronic device is treated as an additional layer on the surface of the skin. With 


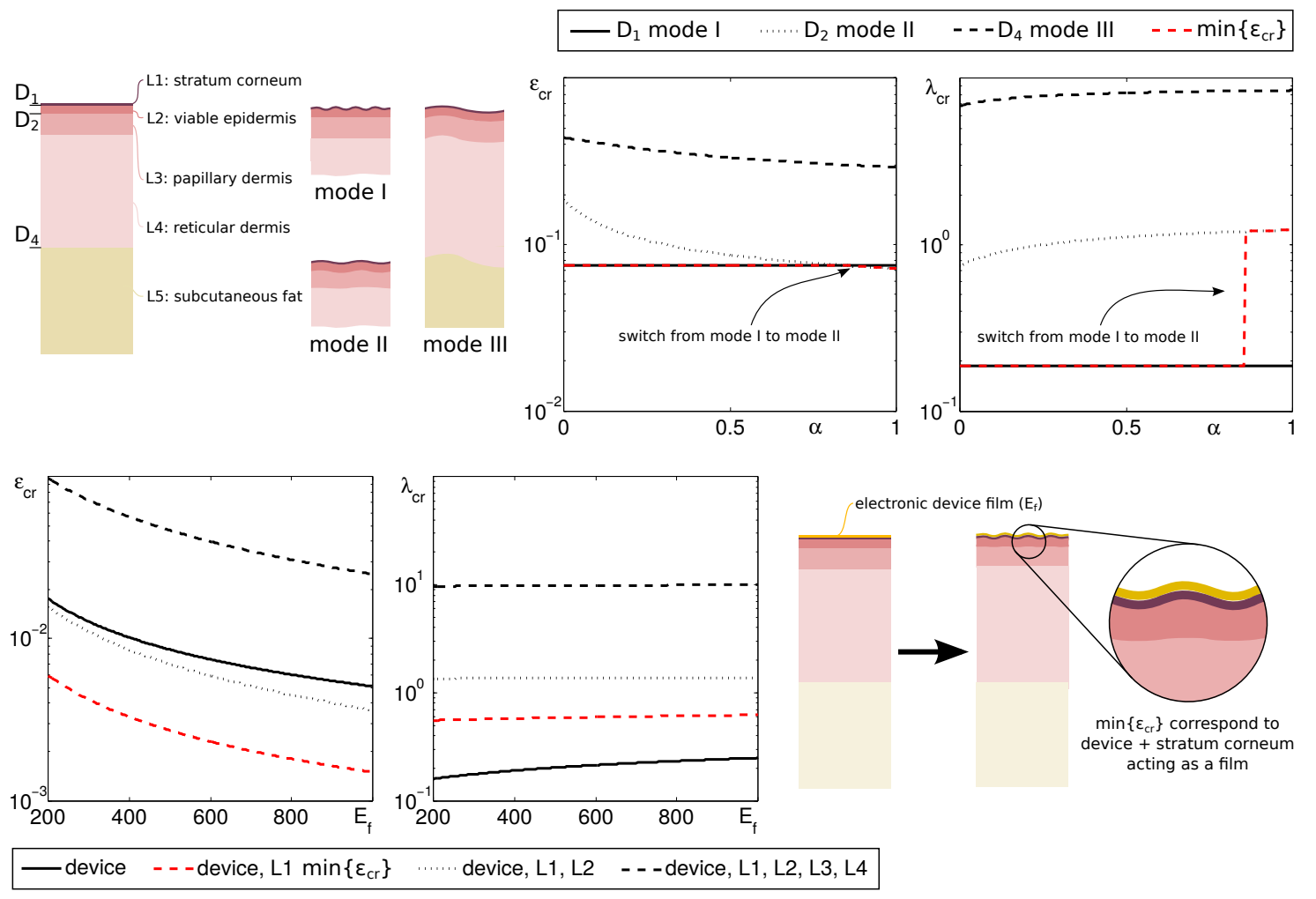

Figure 7: Upper row: wrinkling in a five layer model of human skin. Lower row: wrinkling in a model of human skin with a device adhered to the top. In the upper row, $\alpha$ represents the degree to which the skin has aged where the modulus of each layer varies linearly between "young" and "old" values according to $\alpha$ [35]. Because there is much variability in the literature for mechanical properties of skin [44], the values taken here are order of magnitude approximations. The un-aged moduli $(\alpha=0)$, from top to bottom are $1 \mathrm{MPa}, 0.1 \mathrm{MPa}, 0.05 \mathrm{mPa}, 0.1 \mathrm{MPa}$ and $0.075 \mathrm{MPa}$. The aged moduli are $10 \mathrm{MPa}, 1 \mathrm{MPa}, 0.1 \mathrm{MPa}, 0.2 \mathrm{MPa}, 0.15$ $\mathrm{MPa}$. Changes in skin thickness due to aging were neglected, from top to bottom the thickness of each layer is $0.02 \mathrm{~mm}, 0.08$ $\mathrm{mm}, 0.2 \mathrm{~mm}, 1 \mathrm{~mm}$ and $2 \mathrm{~mm}$ similar to parameters chosen in [35]. In the lower row, skin properties corresponding to $\alpha=0.5$ were chosen while the stiffness of the device $E_{f}$ was varied in units of $M P a$. The device layer is $0.005 \mathrm{~mm}$ thick. Besides the highly intuitive result, that increasing $E_{f}$ results in lower $\varepsilon_{\mathrm{cr}}$ and higher $\lambda_{\mathrm{cr}}$, it is noteworthy that the predicted mode of wrinkling, corresponding to minimum $\varepsilon_{\mathrm{cr}}$ is the device and stratum corneum acting as a composite film while the remainder of the skin acts as a substrate.

our model, we can qualitatively understand what occurs when the device-skin system is subject to compression. Figure 7 compares $\varepsilon_{\mathrm{cr}}$ and $\lambda_{\mathrm{cr}}$ of the device-skin system with different device stiffness to the behavior of the skin alone. The predicted decrease in $\varepsilon_{\mathrm{cr}}$ and increase in $\lambda_{\mathrm{cr}}$ is a highly intuitive result given that the additional layer increases the stiffness of any film or composite film layer that may arise. However, our model also predicts that the first mode of wrinkling to occur will be the system in which the device and stratum corneum act as a composite film. Before applying our model, it was unclear if the lowest value of $\varepsilon_{\text {cr }}$ would correspond to the device acting as a film with the entire skin as a substrate or if layers of the skin would also act as a film. The results of our study are illustrated in Fig. 7 .
Moving forward, the notion that the device and the stratum corneum wrinkle together may contribute to the understanding of buckling delamination in these systems [30, 33, 52, 53]. Though the results presented here are only valid for a given set of parameters, our model has the benefit of being easily implemented and tested for alternative layered device configurations or skin properties.

\section{Conclusion}

In this paper, we outline a procedure for computing the analytical solution for wrinkling instability initiation in multi-layer systems. We begin by presenting our previously developed solution for multi-layer wrinkling 
in systems with a limited number of very thin interfacial layers. Then, we extend this solution to multi-layer systems with an unlimited number of layers and unconstrained layer thickness. Performing this extension has utility in many areas, ranging from stretchable electronics [26, 54], to understanding biological pattern formation [55], to manufacturing biomimetic surface topology [20]. In this paper, we demonstrate the utility of our model for predicting multi-layer wrinkling in epidermal electronics. The configuration of these systems in highly varied and rapidly evolving which further motives our presentation of a general model for multi-layer wrinkling.

\section{Acknowledgments}

This material is based upon work supported by the National Science Foundation Graduate Research Fellowship under Grant No. DGE-114747.

\section{References}

[1] H. G. Allen, Analysis and Design of Structural Sandwich Panels: The Commonwealth and International Library: Structures and Solid Body Mechanics Division, Elsevier, 2013.

[2] Z. Huang, W. Hong, Z. Suo, Evolution of wrinkles in hard films on soft substrates, Physical Review E 70 (2004) 030601

[3] Y. Mei, S. Kiravittaya, S. Harazim, O. G. Schmidt, Principles and applications of micro and nanoscale wrinkles, Materials Science and Engineering R: Reports 70 (3-6) (2010) 209-224.

[4] H. Mei, R. Huang, J. Y. Chung, C. M. Stafford, H. H. Yu, Buckling modes of elastic thin films on elastic substrates, Applied Physics Letters 90 (15) (2007) 1-4.

[5] J. Song, H. Jiang, Z. J. Liu, D. Y. Khang, Y. Huang, J. A. Rogers, C. Lu, C. G. Koh, Buckling of a stiff thin film on a compliant substrate in large deformation, International Journal of Solids and Structures 45 (10) (2008) 3107-3121.

[6] S. Wang, J. Song, D. H. Kim, Y. Huang, J. A. Rogers, Local versus global buckling of thin films on elastomeric substrates, Applied Physics Letters 93 (2) (2008) 3-5.

[7] R. Zhao, T. Zhang, M. Diab, H. Gao, K.-S. Kim, The primary bilayer ruga-phase diagram I: Localizations in ruga evolution, Extreme Mechanics Letters 4 (2015) 76-82.

[8] H. Cheng, Y. Zhang, K. C. Hwang, J. A. Rogers, Y. Huang, Buckling of a stiff thin film on a pre-strained bi-layer substrate, International Journal of Solids and Structures 51 (18) (2014) 3113-3118.

[9] F. Jia, Y.-P. Cao, T.-S. Liu, Y. Jiang, X.-Q. Feng, S.-W. Yu, Wrinkling of a bilayer resting on a soft substrate under in-plane compression, Philosophical Magazine 92 (12) (2012) 1554-1568.

[10] E. Lejeune, A. Javili, C. Linder, Understanding geometric instabilities in thin films via a multi-layer model, Soft Matter doi: 10.1039/c5sm02082d

[11] M. Ben Amar, F. Jia, Anisotropic growth shapes intestinal tissues during embryogenesis., Proceedings of the National Academy of Sciences of the United States of America 110 (26) (2013) 10525-30.
[12] E. Hannezo, J. Prost, J. F. Joanny, Instabilities of monolayered epithelia: Shape and structure of villi and crypts, Physical Review Letters 107 (August) (2011) 1-5.

[13] A. E. Shyer, T. Tallinen, N. L. Nerurkar, Z. Wei, E. S. Gil, D. L. Kaplan, C. J. Tabin, L. Mahadevan, Villification: how the gut gets its villi., Science (New York, N.Y.) 342 (6155) (2013) 2128.

[14] C. Flynn, B. A. O. Mccormack, Finite element modelling of forearm skin wrinkling, Skin Research and Technology 14 (3) (2008) 261-269.

[15] C. Flynn, B. A. O. McCormack, A three-layer model of skin and its application in simulating wrinkling., Computer Methods in Biomechanics and Biomedical Engineering 12 (2) (2009) 125134.

[16] C. Flynn, B. A. O. Mccormack, Simulating the wrinkling and aging of skin with a multi-layer finite element model., Journal of biomechanics 43 (3) (2010) 442-8.

[17] S.-H. Chung, J. H. Lee, J.-H. Yoon, H. K. Lee, K. Y. Seo, Multilayered culture of primary human conjunctival epithelial cells producing MUC5AC., Experimental eye research 85 (2) (2007) 226-33.

[18] M. Eskandari, M. R. Pfaller, E. Kuhl, On the role of mechanics in chronic lung disease, Materials 6 (12) (2013) 5639-5658.

[19] H. E. Jeong, M. K. Kwak, K. Y. Suh, Stretchable, AdhesionTunable Dry Adhesive by Surface Wrinkling, Langmuir 26 (4) (2010) 2223-2226.

[20] J. H. Lee, H. W. Ro, R. Huang, P. Lemaillet, T. a. Germer, C. L. Soles, C. M. Stafford, Anisotropic, hierarchical surface patterns via surface wrinkling of nanopatterned polymer films, Nano Letters 12 (11) (2012) 5995-5999.

[21] A. Schweikart, A. Fery, Controlled wrinkling as a novel method for the fabrication of patterned surfaces, Microchimica Acta 165 (3-4) (2009) 249-263.

[22] J. Y. Chung, A. J. Nolte, C. M. Stafford, Surface wrinkling: A versatile platform for measuring thin-film properties, Advanced Materials 23 (3) (2011) 349-368.

[23] C. Lu, I. Dönch, M. Nolte, A. Fery, Au nanoparticle-based multilayer ultrathin films with covalently linked nanostractures: Spraying layer-by-layer assembly and mechanical property characterization, Chemistry of Materials 18 (26) (2006) 6204-6210.

[24] A. J. Nolte, M. F. Rubner, R. E. Cohen, Determining the Young s Modulus of Polyelectrolyte Multilayer Films via StressInduced Mechanical Buckling Instabilities, Macromolecules 38 (2005) 5367-5370.

[25] A. J. Nolte, R. E. Cohen, M. F. Rubner, A two-plate buckling technique for thin film modulus measurements: Applications to poly electrolyte multilayers, Macromolecules 39 (14) (2006) 4841-4847.

[26] D. Y. Khang, J. a. Rogers, H. H. Lee, Mechanical buckling: Mechanics, metrology, and stretchable electronics, Advanced Functional Materials 19 (10) (2009) 1526-1536.

[27] Y. Sun, W. M. Choi, H. Jiang, Y. Y. Huang, J. A. Rogers, Controlled buckling of semiconductor nanoribbons for stretchable electronics, Nature nanotechnology 1 (3) (2006) 201-207.

[28] K.-I. Jang, S. Y. Han, S. Xu, K. E. Mathewson, Y. Zhang, J.-W. Jeong, G.-T. Kim, R. C. Webb, J. W. Lee, T. J. Dawidczyk, R. H. Kim, Y. M. Song, W.-H. Yeo, S. Kim, H. Cheng, S. I. Rhee, J. Chung, B. Kim, H. U. Chung, D. Lee, Y. Yang, M. Cho, J. G. Gaspar, R. Carbonari, M. Fabiani, G. Gratton, Y. Huang, J. A. Rogers, Rugged and breathable forms of stretchable electronics with adherent composite substrates for transcutaneous monitoring, Nature Communications 5 (2014) 4779.

[29] M. Kaltenbrunner, T. Sekitani, J. Reeder, T. Yokota, K. Kuribara, T. Tokuhara, M. Drack, R. Schwödiauer, I. Graz, S. Bauer- 
Gogonea, S. Bauer, T. Someya, An ultra-lightweight design for imperceptible plastic electronics, Nature 499 (7459) (2013) 458-463.

[30] D.-H. Kim, N. Lu, R. Ma, Y.-S. Kim, R.-H. Kim, S. Wang, J. Wu, S. M. Won, H. Tao, A. Islam, K. J. Yu, T.-i. Kim, R. Chowdhury, M. Ying, L. Xu, M. Li, H.-J. Chung, H. Keum, M. McCormick, P. Liu, Y.-W. Zhang, F. G. Omenetto, Y. Huang, T. Coleman, J. A. Rogers, Epidermal Electronics, Science 333 (September) (2011) 838-844.

[31] J. J. S. Norton, D. S. Lee, J. W. Lee, W. Lee, O. Kwon, P. Won, S.-Y. Jung, H. Cheng, J.-W. Jeong, A. Akce, S. Umunna, I. Na, Y. H. Kwon, X.-Q. Wang, Z. Liu, U. Paik, Y. Huang, T. Bretl, W.-H. Yeo, J. A. Rogers, Soft, curved electrode systems capable of integration on the auricle as a persistent braincomputer interface, Proceedings of the National Academy of Sciences 112 (13) (2015) 201424875.

[32] W. H. Yeo, Y. S. Kim, J. Lee, A. Ameen, L. Shi, M. Li, S. Wang, R. Ma, S. H. Jin, Z. Kang, Y. Huang, J. A. Rogers, Multifunctional epidermal electronics printed directly onto the skin, Advanced Materials 25 (20) (2013) 2773-2778.

[33] S. Wang, M. Li, J. Wu, D.-H. Kim, N. Lu, Y. Su, Z. Kang, Y. Huang, J. A. Rogers, Mechanics of Epidermal Electronics, Journal of Applied Mechanics 79 (3) (2012) 031022.

[34] F. M. Hendriks, D. Brokken, C. W. J. Oomens, D. L. Bader, F. P. T. Baaijens, The relative contributions of different skin layers to the mechanical behavior of human skin in vivo using suction experiments, Medical Engineering and Physics 28 (3) (2006) 259-266.

[35] O. Kuwazuru, J. Saothong, N. Yoshikawa, Evaluation of aging effects on skin wrinkle by finite element method, Journal of Biomechanical Science and Engineering 3 (3) (2008) 368-379.

[36] Biostamp temporary tattoo electronic circuits by MC10, Dezeen Magazine.

[37] C. M. Stafford, B. D. Vogt, C. Harrison, D. Julthongpiput, R. Huang, Elastic moduli of ultrathin amorphous polymer films, Macromolecules 39 (15) (2006) 5095-5099.

[38] Z. Huang, W. Hong, Z. Suo, Nonlinear analyses of wrinkles in a film bonded to a compliant substrate, Journal of the Mechanics and Physics of Solids 53 (9) (2005) 2101-2118.

[39] M. A. Biot, On bending of an infinite beam on an elastic Foundation, Journal of Applied Mathematics and Mechanics 22 (5) (1937) 984-988.

[40] D. Lee, J. R. Barber, M. D. Thouless, Indentation of an elastic half space with material properties varying with depth, International Journal of Engineering Science 47 (2009) 1274-1283.

[41] Y. Cao, J. W. Hutchinson, Wrinkling Phenomena in NeoHookean Film/Substrate Bilayers, Journal of Applied Mechanics 79 (May) (2012) 031019.

[42] M. Diab, T. Zhang, R. Zhao, H. Gao, K.-S. Kim, Ruga mechanics of creasing: from instantaneous to setback creases, Proceedings of the Royal Society A: Mathematical, Physical and Engineering Sciences 469 (2157) (2013) 20120753-20120753.

[43] A. Javili, B. Dortdivanlioglu, E. Kuhl, C. Linder, Computational aspects of growth-induced instabilities through eigenvalue analysis, Computational Mechanics 56 (3) (2015) 405-420.

[44] M. F. Leyva-Mendivil, A. Page, N. W. Bressloff, G. Limbert, A mechanistic insight into the mechanical role of the stratum corneum during stretching and compression of the skin, Journal of the Mechanical Behavior of Biomedical Materials 49 (0) (2015) 197-219.

[45] M. Geerligs, L. van Breemen, G. Peters, P. Ackermans, F. Baaijens, C. Oomens, In vitro indentation to determine the mechanical properties of epidermis, Journal of Biomechanics 44 (6) (2011) 1176-1181.

[46] J.-L. Gennisson, T. Baldeweck, M. Tanter, S. Catheline,
M. Fink, L. Sandrin, C. Cornillon, B. Querleux, Assessment of elastic parameters of human skin using dynamic elastography., IEEE transactions on ultrasonics, ferroelectrics, and frequency control 51 (8) (2004) 980-9.

[47] C. Li, G. Guan, R. Reif, Z. Huang, R. K. Wang, Determining elastic properties of skin by measuring surface waves from an impulse mechanical stimulus using phase-sensitive optical coherence tomography, Journal of The Royal Society Interface 9 (70) (2012) 831-841.

[48] X. L. X. Liang, S. Boppart, Biomechanical properties of in vivo human skin from dynamic optical coherence elastography, IEEE Transactions on Biomedical Engineering 57 (4) (2010) 953959.

[49] J. Welzel, E. Lankenau, R. Birngruber, R. Engelhardt, Optical coherence tomography of the human skin, Journal of the American Academy of Dermatology 37 (6) (1997) 958-963.

[50] F. Hendriks, Mechanical Behaviour of Human Skin in Vivo, Bio-medical Engineering 4 (1969) 322-327.

[51] O. Kuwazuru, K. Miyamoto, N. Yoshikawa, S. Imayama, Skin wrinkling morphology changes suddenly in the early 30s, Skin Research and Technology 18 (4) (2012) 495-503.

[52] H. Cheng, S. Wang, Mechanics of Interfacial Delamination in Epidermal Electronics Systems, Journal of Applied Mechanics 81 (4) (2013) 044501.

[53] H. Mei, R. Huang, Wrinkling and Delamination of Thin Films on Compliant Substrates 2 . Wrinkling Analysis (2013) 1-9.

[54] D.-Y. Khang, H. Jiang, Y. Huang, J. A. Rogers, A Stretchable Form of Single-Crystal, Science 311 (January) (2006) 208-212.

[55] P. Ciarletta, V. Balbi, E. Kuhl, Pattern Selection in Growing Tubular Tissues, Physical Review Letters 113 (24) (2014) 248101. 